\title{
Develop and Evaluate a New and Effective Approach for Predicting Dyslipidemia in Steel Workers
}

OPEN ACCESS

Edited by:

Honghao Gao,

Shanghai University, China

Reviewed by:

Xiaoxian Yang,

Shanghai Second Polytechnic

University, China

Gunasekaran Manogaran,

University of California, Davis,

United States

Jingxia Chen,

Shaanxi University of Science and

Technology, Xi'an, China

Jing Zhang,

Jining Medical University, China

${ }^{*}$ Correspondence:

Juxiang Yuan

gwxyjxb@ncst.edu.cn

Specialty section:

This article was submitted to

Computational Genomics,

a section of the journal

Frontiers in Bioengineering and

Biotechnology

Received: 05 April 2020

Accepted: 30 June 2020

Published: 10 September 2020

Citation

Wu J, Qin S, Wang J, Li J,

Wang H, Li H, Chen Z, Li C, Wang J

and Yuan J (2020) Develop

and Evaluate a New and Effective Approach for Predicting Dyslipidemia

in Steel Workers.

Front. Bioeng. Biotechnol. 8:839.

doi: 10.3389/fbioe.2020.00839

\begin{abstract}
Jianhui Wu ${ }^{1,2}$, Sheng Qin', Jie Wang ${ }^{1}$, Jing Li', Han Wang ${ }^{1}$, Huiyuan Li', Zhe Chen ${ }^{1}$, Chao Li ${ }^{1}$, Jiaojiao Wang ${ }^{1}$ and Juxiang Yuan ${ }^{1,2 *}$

${ }^{1}$ School of Public Health, North China University of Science and Technology, Tangshan, China, ${ }^{2}$ Hebei Province Key Laboratory of Occupational Health and Safety for Coal Industry, North China University of Science and Technology, Tangshan, China
\end{abstract}

The convolutional neural network (CNN) has made certain progress in image processing, language processing, medical information processing and other aspects, and there are few relevant researches on its application in disease risk prediction. Dyslipidemia is a major and modifiable risk factor for cardiovascular disease, early detection of dyslipidemia and early intervention can effectively reduce the occurrence of cardiovascular diseases. Risk prediction model can effectively identify high-risk groups and is widely used in public health and clinical medicine. Steel workers are a special occupational group. Their particular occupational hazards, such as high temperatures, noise and shift work, make them more susceptible to disease than the general population, which makes the risk prediction model for the general population no longer applicable to steel workers. Therefore, it is necessary to establish a new model dedicated to the prediction of dyslipidemia of steel workers. In this study, the physical examination information of thousands of steel workers was collected, and the risk factors of dyslipidemia in steel workers were screened out. Then, based on the data characteristics, the corresponding parameters were set for the convolutional neural network model, and the risk of dyslipidemia in steel workers was predicted by using convolutional neural network. Finally, the predictive performance of the convolutional neural network model is compared with the existing predictive models of dyslipidemia, logistics regression model and BP neural network model. The results show that the convolutional neural network has a good predictive performance in the risk prediction of dyslipidemia of steel workers, and is superior to the Logistic regression model and BP neural network model.

Keywords: deep learning, convolutional neural network, dyslipidemia, steel worker, disease model prediction, model performance comparison

\section{INTRODUCTION}

Dyslipidemia is a chronic noncommunicable disease of lipid metabolism disorder, characterized by increased and/or decreased lipid levels in the blood. With the rapid development of China's economy and the change of life style, cardiovascular disease has become the main death disease of residents (Roth et al., 2017). In recent years, the blood lipid level of Chinese 
population has gradually increased, and the prevalence of dyslipidemia has increased significantly. Evidence demonstrates that dyslipidemia is an independent and modifiable major risk factor for cardiovascular disease, and its level can significantly increase the incidence and mortality of cardiovascular disease (Pikula et al., 2015; Lee et al., 2017). Studies have shown (Miller, 2009; Hendrani et al., 2016; Stevens et al., 2016) that the early detection and management of high-risk groups with dyslipidemia can effectively reduce the incidence of cardiovascular disease, which can reduce the burden of cardiovascular disease and brings great social value.

China has a huge number of steel workers. Steel workers are a special occupational group, whose occupational environment is special, such as high temperature, noise, shift system and other special occupational exposure can cause or affect the occurrence of chronic diseases (Chauhan et al., 2014; Hedén Stahl et al., 2014; Tong et al., 2017; Wu et al., 2019b). Therefore, the prediction model of dyslipidemia in the general population is not suitable for steel workers. In order to improve the quality of life and health status of steel workers, it is urgent to establish a new risk prediction model of dyslipidemia in steel workers.

Logistics regression is a traditional prediction model, which is widely used in the field of disease prediction due to its clear parameter significance and easy to understand outcome indicators (Liu et al., 2018). However, its applicable conditions are relatively strict, which often limits the accuracy of its predictions. BP neural network is a widely used artificial neural network for disease prediction (Yao et al., 2019). Its good nonlinear processing ability and flexible grid structure make it have a good selflearning ability. However, it has a slow learning speed and is liable to fall into local minima, which makes its network promotion ability limited. Convolutional neural network is a kind of feedforward neural network with deep structure and convolution computation. The convolution structure can reduce the memory occupied by the neural network and has strong adaptability. It is good at mining local features of data and extracting global training features and classification, which has some advantages that traditional technologies do not have. In addition, the three key operations of convolution kernel, "local receptive field," weight sharing and pooling, can effectively reduce the number of network parameters, significantly reduce the computational complexity, and alleviate the problem of model overfitting.

Based on thousands of physical examination data of steel workers, we established a convolutional neural network model to predict the risk of dyslipidemia of steel workers, and compared the prediction performance with the existing dyslipidemia prediction model. Overall, our study consists of three contributions:

1. Based on thousands of physical examination data of steel workers, we screened out the risk factors of dyslipidemia of steel workers, which can provide a basis for formulating early prevention strategies for dyslipidemia of steel workers.

2. Combine the characteristics of the data to set the corresponding parameters of the model, and use the convolutional neural network to predict the risk of dyslipidemia in steel workers. We found that the convolutional neural network has a good fit with the physical examination data of steel workers, and has a good prediction performance.

3. Compare the prediction performance of the convolutional neural network model with some of the existing dyslipidemia prediction models and find that the prediction performance of the convolutional neural network model is better. In this way, we can use convolutional neural networks to predict the risk of dyslipidemia of steel workers, so as to achieve the early prevention of dyslipidemia of steel workers and improve the health and quality of life of steel workers.

\section{RELATED WORK}

Disease risk prediction model is a very effective way for early detection of high-risk groups. In recent years, more and more studies on model prediction of dyslipidemia have been conducted, such as Xinghua Yang et al. (2018) established a logistics model of dyslipidemia using a longitudinal database based on Taiwanese MJ health checkups. Chongjian Wang et al. (2012) established an artificial neural network model to identify those at high risk of dyslipidemia in rural adult residents. Xiaoshuai Zhang et al. (2019) used a random forest survival model to predict the risk of dyslipidemia in Chinese Han adults. However, these studies are aimed at the general population, and there are few studies on the risk prediction of dyslipidemia in special occupational populations.

Convolutional neural network has been widely used in medical research and has shown good accuracy and generalization ability (Lee et al., 2018; Lin et al., 2018; Horiuchi et al., 2019; Wu et al., 2019a). However, no one has tried to establish and evaluate the effect of convolutional neural network model on predicting the risk of dyslipidemia in steel workers.

\section{MATERIALS AND METHODS}

\section{Study Population}

This study was a cross-sectional survey. Based on the baseline data of the health effects cohort study of the occupational population in the Beijing-Tianjin-Hebei region, steel workers who had undergone occupational health examinations in a steel group company hospital from March 2017 to June 2017 were selected as the research objects. To be eligible, steel workers must on-the-job for at least 1 year, aged $\leq 60$ years and free from incomplete health examination data. Ultimately, a total of 4655 steel workers were included in the study. All steel workers included in the study received written informed consent. According to the 2016 Chinese guidelines for the management of dyslipidemia in adults (Joint committee for guideline revision, 2018), the steel workers were divided into the dyslipidemia group and the non-dyslipidemia group. Dyslipidemia refers to the total cholesterol (TC) $\geq 6.2 \mathrm{mmol} / \mathrm{L}$, and/or triglyceride $(\mathrm{TG}) \geq 2.3 \mathrm{mmol} / \mathrm{L}$, and/or low-density 
lipoprotein cholesterol (LDL-c) $\geq 4.1 \mathrm{mmol} / \mathrm{L}$, and/or highdensity lipoprotein cholesterol (HDL-c) $<1.0 \mathrm{mmol} / \mathrm{L}$.

\section{Data Collection}

The basic personal information of the steel workers was collected in a one-to-one questionnaire by trained investigators, which mainly included ethnicity, age, gender, education leve, marital status, income, family history of hyperlipidemia, drinking status, smoking status, physical activity, diet, etc. Anthropometric data are measured and collected by doctors and professional trainers in the physical examination center according to the unified standards, which mainly include weight, height, hip circumference, waist circumference, blood pressure, etc. Then the body mass index (BMI) is calculated by dividing the weight

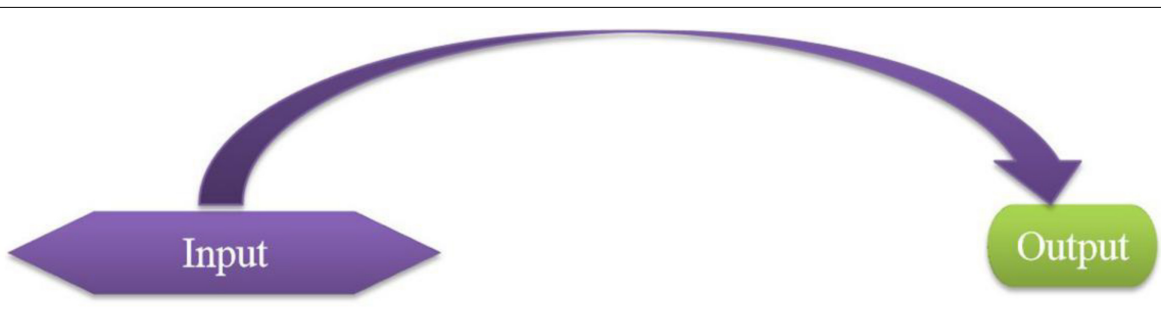

\begin{tabular}{|c|c|c|c|c|c|c|c|}
\hline & Age & $\begin{array}{c}\text { Marital } \\
\text { status } \\
\end{array}$ & Gender & BMI & Education & $\begin{array}{c}\text { Smoking } \\
\text { status } \\
\end{array}$ & \begin{tabular}{|c|c} 
Hyperten- \\
sion \\
\end{tabular} \\
\hline Case 1 & $\mathrm{X}_{1,1}$ & $\mathrm{X}_{1,2}$ & $\mathrm{X}_{1,3}$ & $\mathrm{X}_{1,4}$ & $\mathrm{X}_{1,5}$ & $\mathrm{X}_{1,6}$ & $\mathrm{X}_{1,7}$ \\
\hline Case 2 & $\mathrm{X}_{2,1}$ & $\mathrm{X}_{2,2}$ & $\mathrm{X}_{2,3}$ & $\mathrm{X}_{2,4}$ & $\mathrm{X}_{2,5}$ & $\mathrm{X}_{2,6}$ & $\mathrm{X}_{2,7}$ \\
\hline Case 3 & $\mathrm{X}_{3,1}$ & $\mathrm{X}_{3,2}$ & $\mathrm{X}_{3,3}$ & $\mathrm{X}_{3,4}$ & $\mathrm{X}_{3,5}$ & $\mathrm{X}_{3,6}$ & $\mathrm{X}_{3,7}$ \\
\hline & & & & & & & \\
\hline Case 4655 & $\mathrm{X}_{4655,1}$ & $\mathrm{X}_{4655,2}$ & $\mathrm{X}_{4655,3}$ & $\mathrm{X}_{4655,4}$ & $\mathrm{X}_{4655,5}$ & $\mathrm{X}_{4655,6}$ & $\mathrm{X}_{4655,7}$ \\
\hline
\end{tabular}

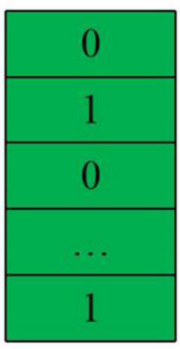

FIGURE 1 | Sample data structure.

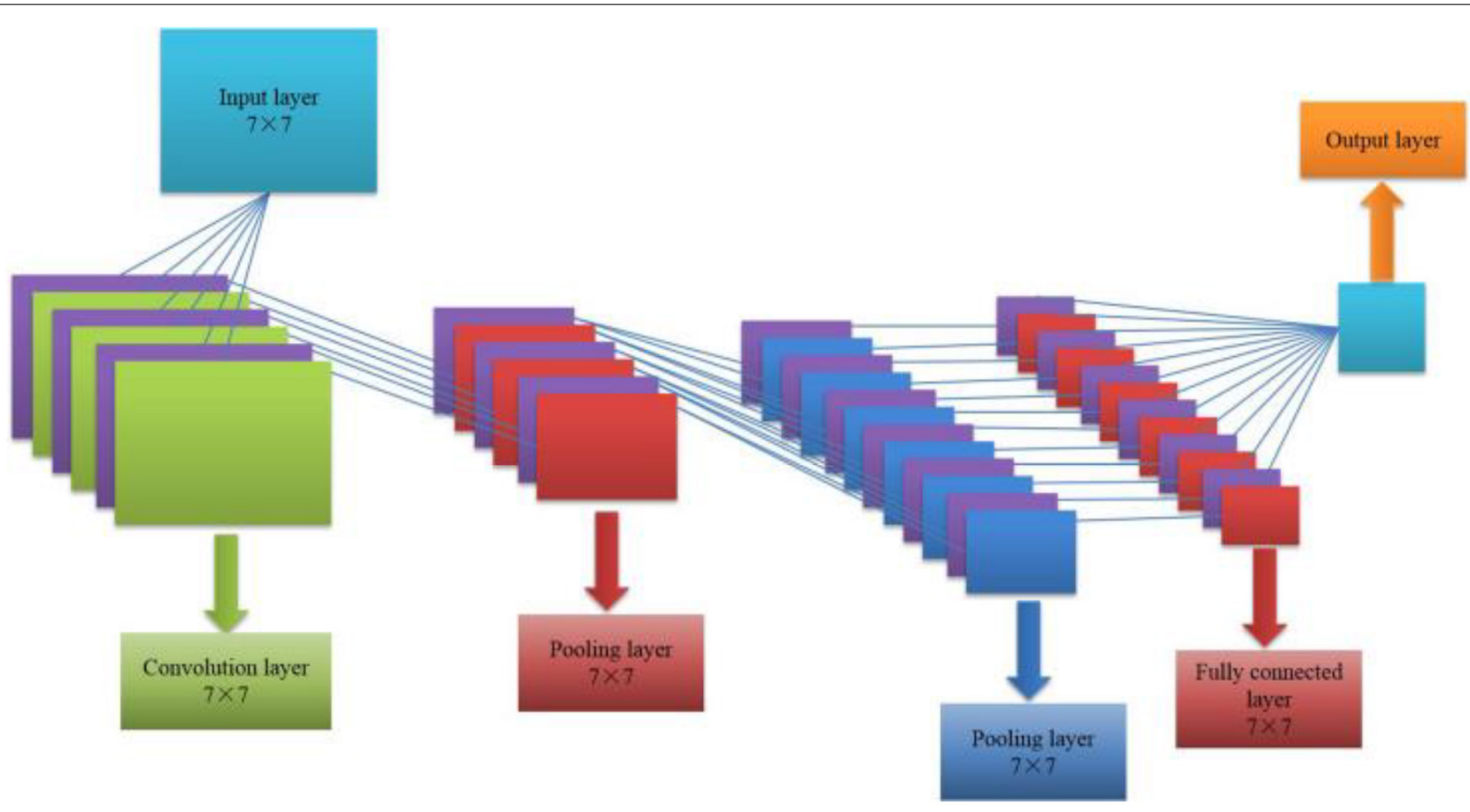

FIGURE 2 | CNN algorithm structure. 
(kg) by the square of height $(\mathrm{m})$, and the waist to hip ratio (WHR) is calculated by dividing the waist circumference $(\mathrm{cm})$ by the hip circumference $(\mathrm{cm})$. Laboratory test data were obtained by analyzing fasting blood samples of steel workers collected by doctors or nurses in the hospital, which mainly included total cholesterol (TC), triglycerides (TG), low-density lipoprotein cholesterol (LDL-C), high-density lipoprotein cholesterol (HDLC), fasting blood glucose, etc. Occupational factors are provided by steel companies, which mainly include high temperature exposure, noise exposure, shift exposure, etc. Hypertension is defined as blood pressure $\geq 140 / 90 \mathrm{mmHg}$ or diagnosed as hypertension by doctors. Diabetes is defined as FPG $\geq 7.0 \mathrm{mmol}$ / $\mathrm{L}$ or diagnosed as diabetes by doctors.

\section{Model Independent Variable Filtering Method}

We established an Excel database based on questionnaires and physical examination data, and screened out independent variables of risk factors for dyslipidemia of steel workers for model prediction. Measurement data was presented as $\bar{X} \pm$ $S$ for normal distribution or $\mathrm{M}(\mathrm{P} 25, \mathrm{P} 75)$ for non-normal distribution, and we used $t$-test or the rank sum test for comparison between groups, respectively. The classification data was expressed by numbers and percentages, and the comparison between groups was performed by Chi-square test. The rank data was presented by numbers and composition ratio, and the rank sum test was used for inter group comparison. Unconditional Logistic regression analysis was used for multivariate analysis of influencing factors. Differences were deemed significant when $p<0.05$. Factors influencing dyslipidemia of steel workers were screened out by univariate analysis and multi-factor logistics regression analysis. In order to avoid the influence of data multicollinearity, the screened influencing factors were diagnosed by multicollinearity. Combined with expert consultation and literature inquiry to determine the appropriate model independent variables. The statistical analysis was performed by SPSS 25.0. ROC curves were drawn using MedCalc.

\section{The Construction of Sample Set}

After screening (the screening results will be introduced later), a total of 4655 steel workers' physical examination data constitute the sample set, as shown in Figure 1. There are seven independent variables, and the output target value is the presence or absence of dyslipidemia (Dyslipidemia is represented by 1 and nondyslipidemia is represented by 0). Meanwhile, 4655 sample data were randomly assigned into $70 \%$ training set $(n=3258), 20 \%$ verification set $(n=931)$, and $10 \%$ test set $(n=466)$.

\section{Convolutional Neural Network Configuration}

Convolutional neural network is an important algorithm in the field of deep learning, including five parts of input layer, convolution layer, activation function, pooling layer and fully connected layer. It continuously adjusts the bias and connection weights between various neurons by combining forward propagation of information and backward propagation of error (Arun et al., 2018; Keshari et al., 2018). Its algorithm structure is shown in Figure 2.

To predict whether steel workers are dyslipidemia by convolutional neural network, setting reasonable complex structure is an important premise to ensure the accuracy of the prediction model. According to the characteristics of the collected data, the network structure of convolutional neural network model designed is shown in Figure 3. We set up 1 input layer, 3 convolution layers, 3 pooling layers, 1 fully connected layer and 1 output layer in convolutional neural network. The size of the convolution kernel set by the convolution layer $1 \sim 3$ is $2 \times 2$, and the number of convolution nuclei is 20 . All the three poolings are maximized sampling (Xu et al., 2015; Iqbal et al., 2018), the core size is $2 \times 2$. The activation functions are all Relu functions. The number of neurons in the whole connective layer is 25 .

\section{Convolutional Neural Network Algorithm Solution}

In this paper, we use the data of thousands of physical examination questionnaires of steel workers and convolution neural network algorithm to analyze and predict whether individuals have dyslipidemia.

A convolutional neural network for data processing rules that input data will pass through one or more hidden layers. In the hidden layer, each data is assigned a weight and bias, so the input data is assigned a new output value. If these new output

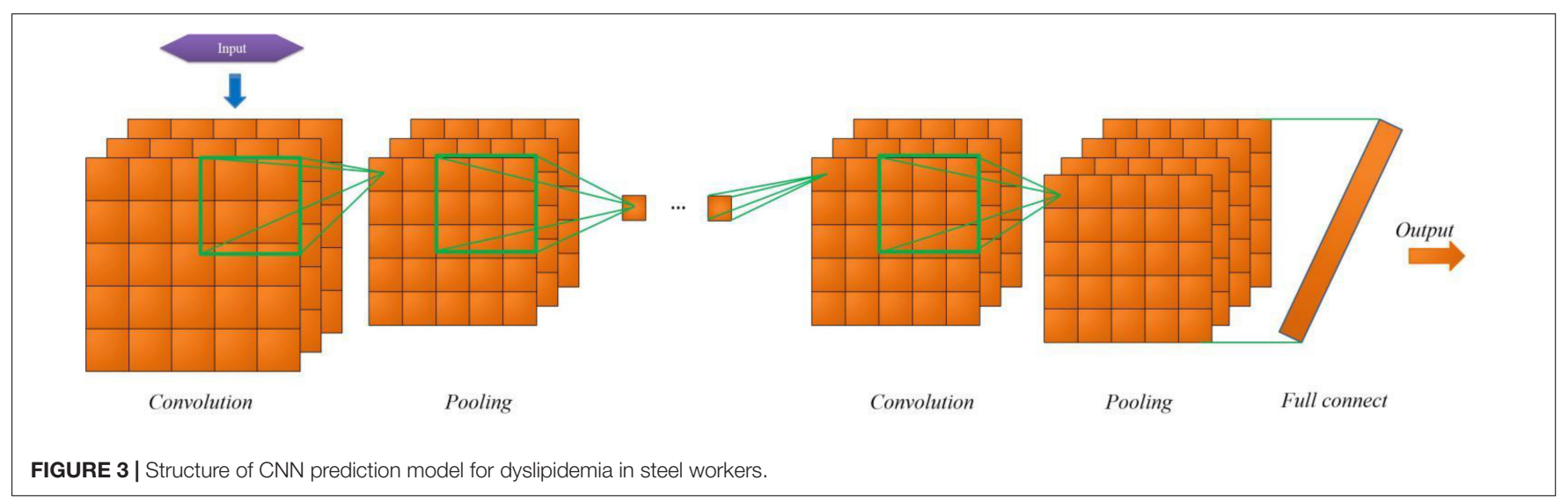




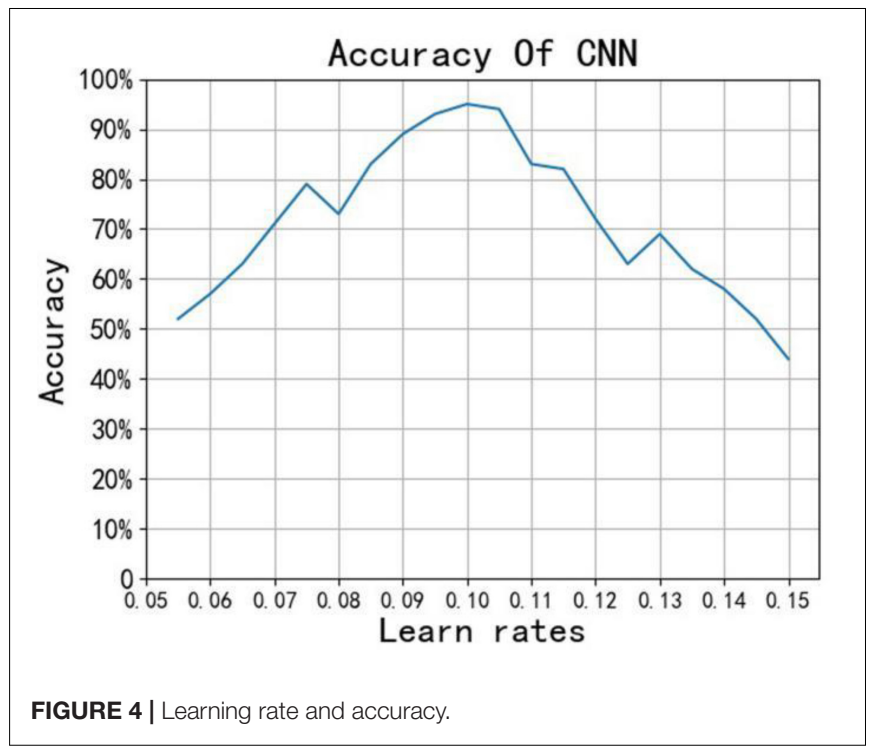

values do not meet expectations, they are also assigned new weights and bias, and the process is repeated to produce the final output. The process mainly includes forward propagation and backward propagation.

The forward propagation calculation formula of convolutional neural network is:

$$
\begin{gathered}
a_{j}^{(l)}=f\left(u^{l}\right) \\
u^{l}=W^{l} a^{(l-1)}+b^{l}
\end{gathered}
$$

Where $a_{j}^{(l)}$ represents the output of layer $l, W^{l}$ represents weights, $b^{l}$ represents biases, $f$ is the activation function.

Since the feature map input in the forward propagation of the convolutional layer is convolved with the convolution kernel, the forward propagation formula of the $j$-th convolution kernel in the $l$-th layer of the convolutional neural network is as follows:

$$
a_{j}^{(l)}=f\left(\sum_{i \in N_{j}} a_{j}^{(l-1)} \cdot k_{i j}^{(l)}+b_{j}^{(l)}\right)
$$

Where $k$ is the convolution kernel, $a_{j}^{(l-1)}$ is the output of the $j$-th convolution kernel of the $l-1$ layer, $N_{j}$ is a choice of input features. $b_{j}^{(l)}$ is the bias Shared by each convolution layer, $f$ is the activation function of the convolution layer.

The forward propagation of the pooling layer requires the pooling calculation of the input features and then other calculations. The calculation formula of the pooling layer is as follows:

$$
a_{j}^{(l)}=f\left(\beta_{j}^{(l)} \text { pooling }\left(a_{j}^{(l-1)}\right)+b_{j}^{(l)}\right)
$$

Where $a_{j}^{(l)}$ is the result of pooling the $j$-th characteristic map

\begin{tabular}{|c|c|c|c|c|}
\hline \multirow[t]{2}{*}{ Variable } & \multicolumn{2}{|c|}{ Dyslipidemia $N(\%) / M(\mathrm{P} 25, \mathrm{P} 75)$} & \multirow[t]{2}{*}{$\chi^{2} / z$} & \multirow[t]{2}{*}{$p$} \\
\hline & No $(N=2860)$ & Yes $(N=1795)$ & & \\
\hline Age & $46(38,50)$ & $46(39,50)$ & 0.739 & 0.46 \\
\hline Gender & & & 54.494 & $<0.001$ \\
\hline Male & 2549 (89.1) & $1711(95.3)$ & & \\
\hline Female & $311(10.9)$ & $84(4.7)$ & & \\
\hline Nation & & & 1.834 & 0.176 \\
\hline Han & 2801 (97.9) & $1747(97.3)$ & & \\
\hline Other & $59(2.1)$ & $48(2.7)$ & & \\
\hline Marital status & & & 2.0046 & $<0.001$ \\
\hline Unmarried & $119(4.2)$ & $34(1.9)$ & & \\
\hline Married & $2670(93.4)$ & $1702(94.8)$ & & \\
\hline Other & $71(2.5)$ & $59(3.3)$ & & \\
\hline Education & & & 18.67 & $<0.001$ \\
\hline $\begin{array}{l}\text { Elementary and } \\
\text { below }\end{array}$ & $37(1.3)$ & $18(1.0)$ & & \\
\hline $\begin{array}{l}\text { Middle and high } \\
\text { school }\end{array}$ & $2142(74.9)$ & $1408(79.4)$ & & \\
\hline $\begin{array}{l}\text { Junior college and } \\
\text { undergraduate }\end{array}$ & $639(22.3)$ & $363(20.2)$ & & \\
\hline $\begin{array}{l}\text { Graduate and } \\
\text { above }\end{array}$ & $42(1.5)$ & $6(0.3)$ & & \\
\hline Monthly income & $6000(4000,7000)$ & $5000(4000,7000)$ & 0.259 & 0.796 \\
\hline $\begin{array}{l}\text { Family history of } \\
\text { hyperlipidemia }\end{array}$ & & & 0.976 & 0.323 \\
\hline No & 2724 (95.2) & 1698 (94.6) & & \\
\hline Yes & $136(4.8)$ & $97(5.4)$ & & \\
\hline Smoking status & & & 93.918 & $<0.001$ \\
\hline No smoking & $1374(48.0)$ & 605 (33.7) & & \\
\hline Quit smoking & $149(5.2)$ & $105(5.8)$ & & \\
\hline smoking & $1337(46.7)$ & $1085(60.4)$ & & \\
\hline Drinking situation & & & 11.509 & 0.003 \\
\hline No drinking & $1762(61.6)$ & 1016 (56.6) & & \\
\hline Quit drinking & $58(2.0)$ & $40(2.2)$ & & \\
\hline Drinking & $1040(36.4)$ & 739 (41.2) & & \\
\hline Physical activity & & & 0.247 & 0.884 \\
\hline Mild & $616(21.5)$ & $378(21.1)$ & & \\
\hline Moderate & $1233(43.1)$ & 786 (43.8) & & \\
\hline Severe & 1011 (35.3) & $631(35.2)$ & & \\
\hline High fat diet score & $12(11,13)$ & $12(11,13)$ & 0.916 & 0.36 \\
\hline $\begin{array}{l}\text { Vegetable Fruit } \\
\text { Score }\end{array}$ & $6(6,7)$ & $6(5,7)$ & 1.748 & 0.08 \\
\hline $\mathrm{BMl}$ & $24.9(22.7,27.2)$ & $26.7(24.5,29.1)$ & 17.277 & $<0.001$ \\
\hline WHR & $0.875(0.831,0.917)$ & $0.901(0.862,0.939)$ & 13.4 & $<0.001$ \\
\hline Diabetes & & & 10.448 & 0.001 \\
\hline No & $2751(96.2)$ & $1690(94.2)$ & & \\
\hline Yes & $109(3.8)$ & $105(5.8)$ & & \\
\hline Hypertension & & & 34.381 & $<0.001$ \\
\hline No & $2480(86.7)$ & 1441 (80.3) & & \\
\hline Yes & $380(13.3)$ & $354(197)$ & & \\
\hline Shift work & & & 6.276 & 0.043 \\
\hline Never shift & $500(17.5)$ & 269 (15.0) & & \\
\hline Once shifts & $509(17.8)$ & $306(17.0)$ & & \\
\hline Now shifts & $1851(64.7)$ & $1220(68.0)$ & & \\
\hline Occupation noise & & & 3.02 & 0.082 \\
\hline No & 2279 (79.7) & $1392(77.5)$ & & \\
\hline Yes & $581(20.3)$ & $403(22.5)$ & & \\
\hline $\begin{array}{l}\text { Occupation high } \\
\text { temperature }\end{array}$ & & & 7.288 & 0.007 \\
\hline No & $2373(83.0)$ & $1433(79.8)$ & & \\
\hline Yes & $487(17.0)$ & 362 (20.2) & & \\
\hline
\end{tabular}
of the $l$-th convolution. Pooling is the pooling operation, $\beta_{j}^{(l)}$ is
TABLE 1 | Comparison of baseline characteristics of dyslipidemia and non-dyslipidemia patients in steel workers. 
TABLE 2 | Multicollinearity diagnostic table.

\begin{tabular}{llc}
\hline & \multicolumn{2}{c}{ Collinearity statistics } \\
\cline { 2 - 3 } & Tolerance & VIF \\
\hline (Constant) & & \\
Age & 0.711 & 1.407 \\
Marital status & 0.91 & 1.099 \\
Gender & 0.873 & 1.146 \\
BMl & 0.936 & 1.069 \\
WHR & 0.979 & 1.021 \\
Education & 0.758 & 1.319 \\
Smoking status & 0.849 & 1.178 \\
Drinking situation & 0.88 & 1.136 \\
Diabetes & 0.966 & 1.035 \\
Hypertension & 0.91 & 1.098 \\
Shift work & 0.966 & 1.035 \\
Occupation high temperature & 0.991 & 1.009 \\
\hline
\end{tabular}

the multiplicative bias of the pooling layer, and $b_{j}^{(l)}$ is the additive bias of the pooling layer.

The back propagation of convolutional neural network. Suppose that the loss function $J_{m s e}$ defined as the convolution neural network is the mean square error, and the formula is as follows:

$$
J_{m s e}=\frac{1}{2} \sum_{i=1}\left(Y_{i}-y_{i}\right)^{2}
$$

Where $Y_{i}$ is the actual value and $y_{i}$ is the output value.

Backpropagation of the fully connected layer in convolutional neural network is obtained by BP algorithm. For the convolution layer of the convolution neural network, if the next layer of the convolution layer $l$ is the fully connected layer, then the sensitivity $\delta_{j}^{(l)}$ of the $j$-th convolution kernel can be obtained by the BP algorithm. If it is the pooling layer, then the calculation formula of the error sensitivity is:

$$
\delta_{j}^{(l)}=\beta_{j}^{(l+1)}\left(f^{\prime}\left(u_{j}^{(l)}\right) \circ u p\left(\delta_{j}^{(l+1)}\right)\right)
$$

Where $\beta_{j}^{(l+1)}$ represents the multiplier bias of the corresponding pooling layer, and up represents the anti-pooling operation. After the error sensitivity of the convolution layer is obtained, the convolution kernel and bias of the convolution layer are updated, and the formula is as follows:

$$
\begin{aligned}
& \frac{\partial J_{m s e}}{\partial k_{i j}^{(l)}}=\sum_{m, n} \delta_{j}^{(l)} p_{j}^{(l-1)} \\
& \frac{\partial J_{m s e}}{\partial b_{j}}=\sum_{m, n}\left(\delta_{j}^{(l)}\right)_{m, n}
\end{aligned}
$$

Where $p_{j}^{(l-1)}$ is the value of $a_{j}^{(l-1)}$ multiplied by each element of the convolution kernel $k_{i j}^{(l)}, m$ and $n$ are the location information of the element in the input feature.

Similarly, the pooling layer is similar to the convolution layer. When the pooling layer is followed by the fully connected layer, the error sensitivity can be obtained by BP algorithm. When the pooling layer is the convolution layer, the error sensitivity is:

$$
\delta_{j}^{(l)}=f^{\prime}\left(u_{j}^{(l)}\right) \circ \operatorname{conv} 2\left(\delta_{j}^{(l+1)}, \operatorname{rot} 180\left(k_{j}^{l+1}\right), ' f u l l^{\prime}\right)
$$

Where conv2 represents the convolution calculation, rot 180 represents the rotation of the matrix by 180 degrees, and full represents the missing data in the matrix replaced by 0 . After the error sensitivity of the pooling layer is obtained, the gradient calculation formula of $b_{j}^{(l)}$ and $\beta_{j}^{(l)}$ is as follows:

$$
\begin{gathered}
\frac{\partial J_{m s e}}{\partial b_{j}}=\sum_{m, n}\left(\delta_{j}^{(l)}\right)_{m, n} \\
\frac{\partial J_{m s e}}{\partial \beta_{j}}=\sum_{m, n}\left(\delta_{j}^{(l)} \circ \text { pooling }\left(a_{j}^{(l-1)}\right)\right)_{m, n}
\end{gathered}
$$

\section{Platform and Parameter Settings}

In this paper, TensorFlow modules in Python are used to

\begin{tabular}{|c|c|c|c|c|c|c|c|c|}
\hline \multirow[t]{2}{*}{ Variable } & \multirow[t]{2}{*}{ B } & \multirow[t]{2}{*}{ S.E. } & \multirow[t]{2}{*}{ Wald } & \multirow[t]{2}{*}{ df } & \multirow[t]{2}{*}{ Sig. } & \multirow[t]{2}{*}{$\operatorname{Exp}(B)$} & \multicolumn{2}{|c|}{ 95\% C.I. for $\operatorname{Exp}(B)$} \\
\hline & & & & & & & Lower & Upper \\
\hline \multicolumn{9}{|l|}{ Marital status (others) } \\
\hline Unmarried & -0.963 & 0.287 & 11.285 & 1 & 0.001 & 0.382 & 0.218 & 0.67 \\
\hline Gender (female) & -0.479 & 0.137 & 12.193 & 1 & 0 & 0.619 & 0.473 & 0.81 \\
\hline $\mathrm{BMl}$ & 0.13 & 0.009 & 198.068 & 1 & 0 & 1.139 & 1.118 & 1.159 \\
\hline \multicolumn{9}{|l|}{ Education (graduate and above) } \\
\hline Middle and high school & 1.072 & 0.452 & 5.612 & 1 & 0.018 & 2.921 & 1.203 & 7.091 \\
\hline Junior college and undergraduate & 1.035 & 0.452 & 5.253 & 1 & 0.022 & 2.815 & 1.162 & 6.821 \\
\hline Smoking status (smoking) & & & 44.924 & 2 & 0 & & & \\
\hline No smoking & -0.473 & 0.071 & 44.821 & 1 & 0 & 0.623 & 0.542 & 0.716 \\
\hline Hypertension & 0.187 & 0.088 & 4.521 & 1 & 0.033 & 1.206 & 1.015 & 1.434 \\
\hline
\end{tabular}
construct the convolutional neural network model. TensorFlow is fully open source and available to anyone with minimal device configuration requirements. It can run models automatically on all platforms, from mobile phones, a single CPU/GPU, to distributed systems consisting of hundreds of GPU CARDS.

TABLE 3 | Multivariate logistics regression analysis of risk factors of dyslipidemia in steel workers. 


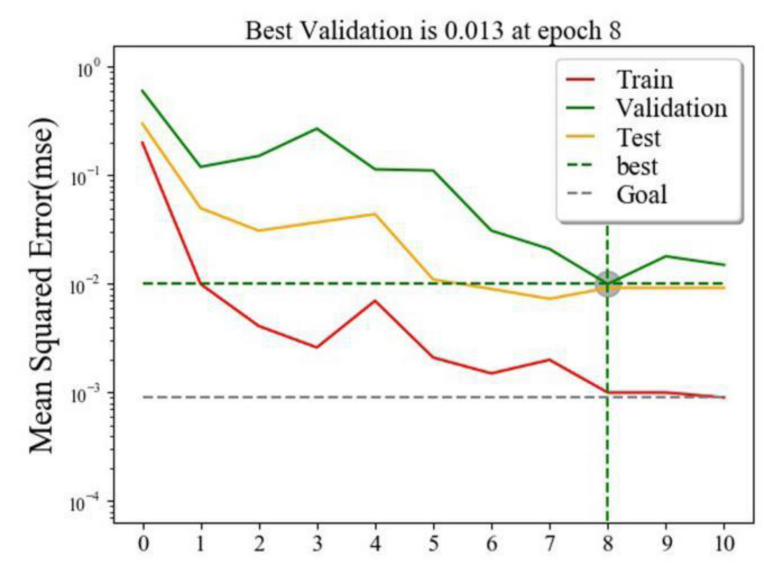

FIGURE 5 | Effect error graph of CNN learning.

We use the random initialization function to set the weight and bias. The smaller the learning rate, the longer the model takes to converge, but it can improve the accuracy of the model.
In order to find the best learning rate of convolutional neural network, we first use random function in Python to initialize the learning rate at random, and then use Python to traverse different learning rates in steps of 0.01 . Finally, use Matplotlib module to make some corresponding images as shown in Figure 4. It can be seen that when the learning rate is about 0.1 , the accuracy is the highest. Therefore, choosing 0.1 as the learning rate can make the convolutional neural network achieve better prediction effect.

\section{Performance Metrics}

In this paper, five performance metrics including accuracy, sensitivity, specificity, F1-score and ROC curve were selected to evaluate the performance of the convolutional neural network model. Meanwhile, the prediction performance of training set and test set of convolutional neural network model, Logistics regression model and BP neural network model was compared. The calculation method of the above metrics are as follows:

$$
\begin{aligned}
& \text { Sensitivity }=\frac{\mathrm{TP}}{\mathrm{TP}+\mathrm{FN}} \\
& \text { Specificity }=\frac{\mathrm{TN}}{\mathrm{TN}+\mathrm{FP}}
\end{aligned}
$$
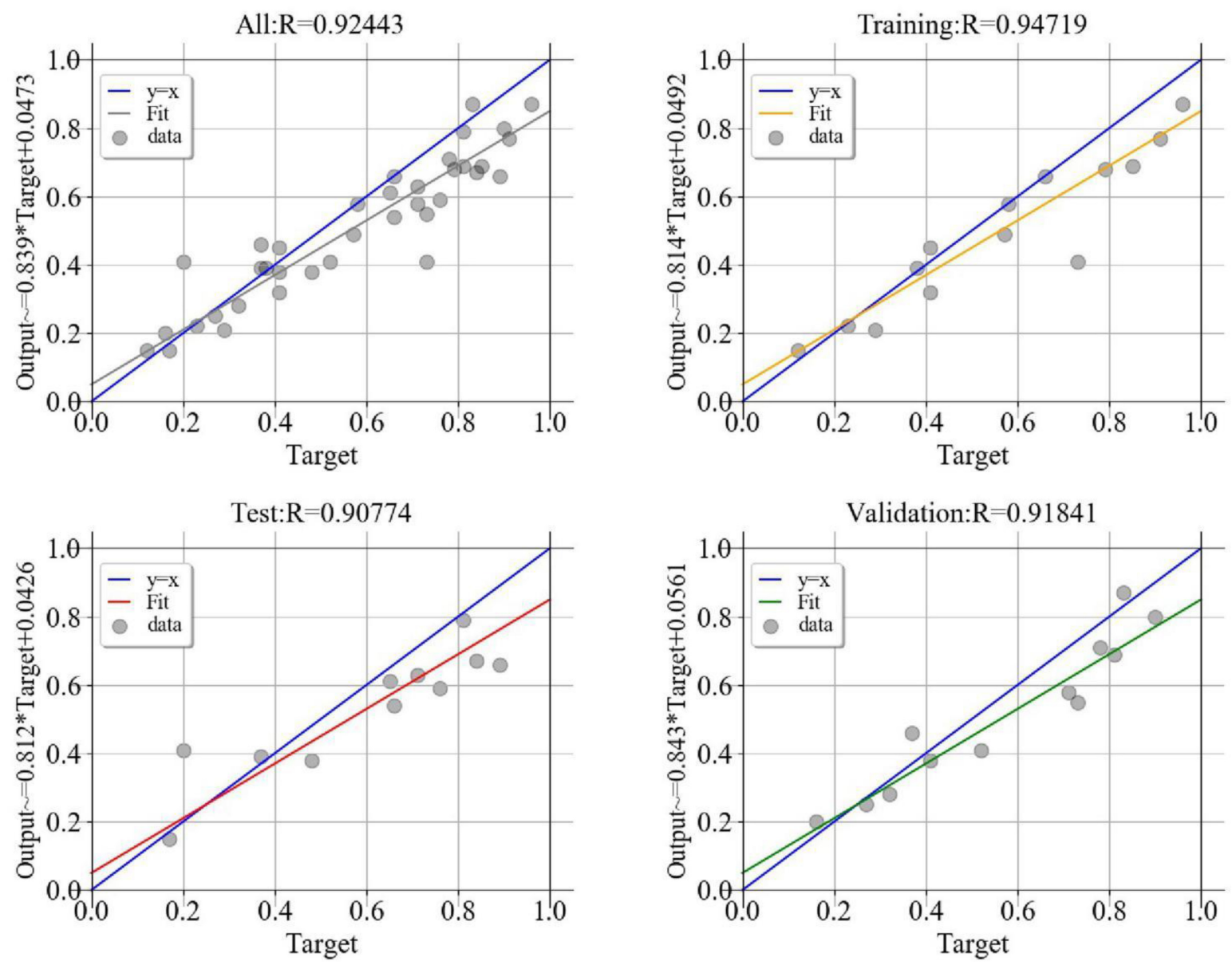

FIGURE 6 | CNN model goodness of fit test chart. 
TABLE 4 | Comparison of performance metrics of each model.

\begin{tabular}{|c|c|c|c|c|c|c|c|c|}
\hline \multirow[t]{2}{*}{ Model } & \multicolumn{4}{|c|}{ Training set } & \multicolumn{4}{|c|}{ Test set } \\
\hline & Sensitivity (\%) & Specificity (\%) & Accuracy (\%) & F1 score & Sensitivity (\%) & Specificity (\%) & Accuracy (\%) & F1 score \\
\hline Logistics & 72.45 & 76.47 & 74.92 & 0.69 & 71.11 & 70.30 & 70.6 & 0.65 \\
\hline BP neural network & 86.7 & 88.96 & 88.09 & 0.85 & 81.11 & 83.57 & 82.62 & 0.78 \\
\hline CNN & 93.23 & 95.65 & 94.72 & 0.93 & 90.00 & 91.26 & 90.77 & 0.88 \\
\hline
\end{tabular}

TABLE 5 | Performance metrics of convolutional neural network.

\begin{tabular}{lccc}
\hline $\begin{array}{l}\text { Performance } \\
\text { metrics }\end{array}$ & Training set & Test set & Validation set \\
\hline Sensitivity (\%) & 93.23 & 90.00 & 89.97 \\
Specificity (\%) & 95.65 & 91.26 & 93.01 \\
Accuracy (\%) & 94.72 & 90.77 & 91.84 \\
F1 score & 0.93 & 0.91 & 0.89 \\
AUC (95\% Cl) & $0.944(0.936-0.952)$ & $0.906(0.876-0.931)$ & $0.915(0.895-0.932)$ \\
\hline
\end{tabular}

$$
\begin{gathered}
\text { Accuracy }=\frac{\mathrm{TP}+\mathrm{TN}}{\mathrm{TP}+\mathrm{FP}+\mathrm{TN}+\mathrm{FN}} \\
F_{1}=2 \cdot \frac{\text { precision } \cdot \text { recall }}{\text { precision }+ \text { recall }} \\
\text { precision }=\frac{\mathrm{TP}}{\mathrm{TP}+\mathrm{FP}} \\
\text { recall }=\frac{\mathrm{TP}}{\mathrm{TP}+\mathrm{FN}}
\end{gathered}
$$

$\mathrm{TP}$ represents true positions, $\mathrm{TN}$ represents true negatives, FP represents false positions, FN represents false negatives. Sensitivity reflects the model's ability to find patients, specificity reflects the model's ability to find non-patients, and accuracy represents the model's overall predictive ability. The F1 score is a harmonic average of the accuracy and recall rates and is used as a final measurement. In addition, ROC curves and AUC are often used to test the balance between true and false positive rates.

\section{RESULTS}

\section{Baseline Characteristics}

A total of 4655 subjects were included in this study, including 1795 cases of dyslipidemia (38.56\%) and 2860 cases of nondyslipidemia (61.43\%). The characteristics of baseline data and the results of univariate analysis are shown in Table $\mathbf{1}$. Univariate analysis showed that there were statistically significant differences $(p<0.05)$ between the dyslipidemia group and the non-dyslipidemia group in gender, educational level, marital status, smoking status, drinking situation, hypertension, diabetes, BMI, waist-to-hip ratio, shift work, and occupational high temperature. Unexpectedly, no significant differences $(p>0.05)$ were observed in ethnicity, age, income, diet, physical activity, family history of hyperlipidemia and occupation noise between the two groups.

\section{Independent Variable Selection}

The significant variables of univariate analysis were used for multicollinearity diagnosis, and age as an influential factor of disease was also included in the analysis. The results show (Table 2) Tolerance $>0.1$ and VIF $<10$, so there is no multicollinearity among the variables. Then, these variables were analyzed by multivariate unconditional logistic regression. The results showed (Table 3) that marital status and educational level were the influencing factors of dyslipidemia. Meanwhile, hypertension is a risk factor for dyslipidemia, male workers have lower risk than female workers, the steel workers who don't smoke have a lower risk. The higher the BMI, the higher the risk of dyslipidemia. Literature supports (Ni et al., 2015; Pereira et al., 2015; Qi et al., 2015) that age is an influential factor of dyslipidemia, so age was included in the model as an independent variable. Finally, according to factor analysis, literature inquiry and expert consultation, seven independent variables were selected to enter the model. The seven independent variables are age, gender, marital status, educational, BMI, smoking status and hypertension.

\section{Convolutional Neural Network Model Results}

The effect error chart (Figure 5) of the dyslipidemia convolutional neural network prediction model shows that the minimum verification error is 0.013 when training in step 8. The goodness of fit test results of the convolutional neural network prediction model for dyslipidemia (Figure 6) show that the training set is 0.974 , the verification set is 0.918 , and the test set is 0.908 . The performance metrics of the convolutional neural network model of dyslipidemia in steel workers are shown in Table 4. The sensitivity is 93.23, 90.00 , and $89.97 \%$ in training set, test set and verification set, respectively. The specificity is 95.65, 91.26, and $93.01 \%$ in training set, test set and verification set, respectively. The accuracy is $94.72,90.77$, and $91.84 \%$ in training set, test set and verification set, respectively. The F1 score is $0.93,0.91$, and 0.89 in training set, test set and verification set, respectively. The AUC (95\% CI) is 0.944 (0.936-0.952), 0.906 (0.876$0.931)$ and $0.915(0.895-0.932)$ in training set, test set and verification set, respectively. The above results show that the convolutional neural network model is very suitable for the physical examination data of steel workers with dyslipidemia. In addition, the convolutional neural network model has a good ability to find patients with dyslipidemia and non-dyslipidemia, and has high prediction accuracy. 


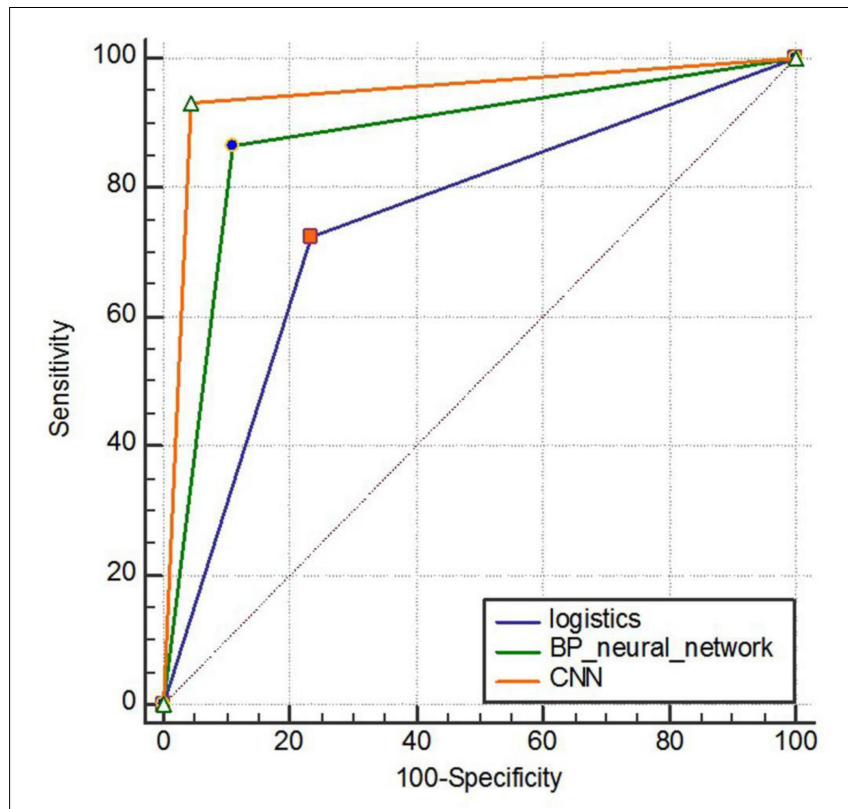

FIGURE 7 | ROC curve comparison of three model training sets.

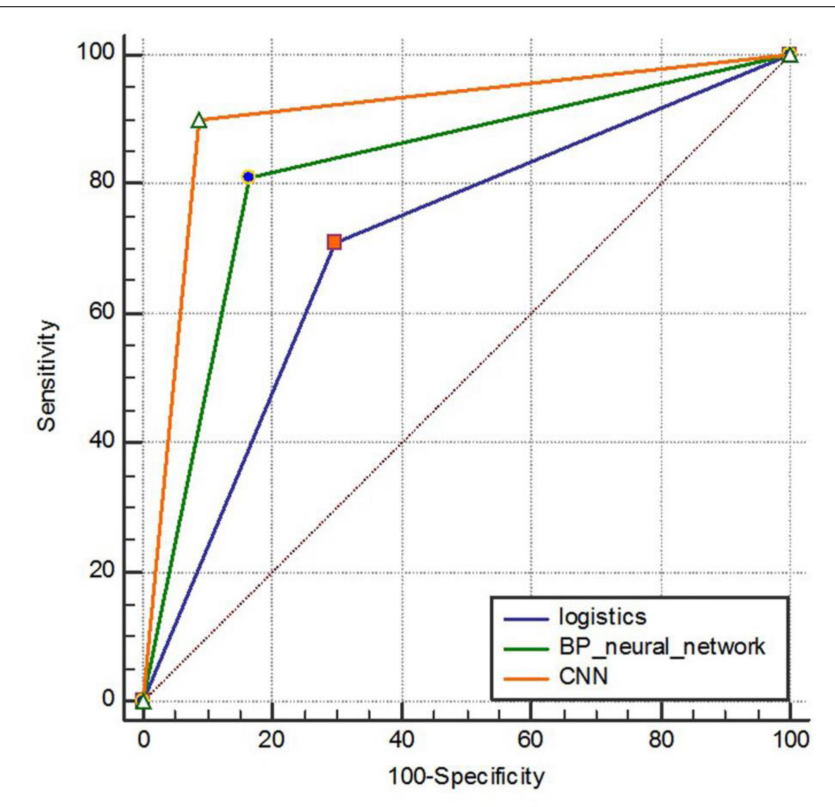

FIGURE 8| ROC curve comparison of three model test sets.

\section{Model Effect Comparison}

We compared the prediction performance of the convolutional neural network model for dyslipidemia in steel workers with that of the Logistics regression model and BP neural network model. The comparison results of performance metrics are shown in Table 5.

Predictive performance results of three model training sets samples. The sensitivity of the logistic regression model, BP neural network model and convolutional neural network model is $72.45 \%, 86.7 \%$ and $92.23 \%$, respectively. The specificity is 76.47 , 88.96 , and $95.65 \%$, respectively. The accuracy is $74.92,88.09$, and $94.72 \%$, respectively. The F1 score is $0.69,0.85$, and 0.93 , respectively. The area under the ROC curve is shown in Figure 7, and the AUC (95\%CI) is 0.745 (0.729-0.760), 0.878 (0.867$0.889)$, and $0.944(0.936-0.952)$, respectively, with statistically significant differences $(P<0.001)$.

Predictive performance results of three model test sets samples. The sensitivity of the logistic regression model, BP neural network model and convolutional neural network model is $71.11,81.11$, and $90.00 \%$, respectively. The specificity is 70.30 , 83.57 , and $91.26 \%$, respectively. The accuracy is $70.60,82.62$, and $90.77 \%$, respectively. The F1 score is $0.65,0.78$ and 0.88 , respectively. The area under the ROC curve is shown in Figure 8, and the AUC (95\%CI) is 0.707 (0.663-0.748), 0.823 (0.786$0.857)$, and $0.906(0.876-0.931)$, respectively, with statistically significant differences $(P<0.001)$.

In combination with the above performance metrics, in the prediction of dyslipidemia of steel workers, the convolutional neural network is optimal in terms of sensitivity, specificity, accuracy, F1 score and AUC. Therefore, in the prediction of dyslipidemia in steel workers, the convolutional neural network has better prediction performance.

\section{CONCLUSION}

In this work, we constructed a convolutional neural network model to predict dyslipidemia in steel workers, a special occupational group. At the beginning, we screened the data and found out the risk factors for dyslipidemia in steel workers to construct a prediction model. Subsequently, we tested the fitting degree of the model and data, and the goodness of fit in the training set, test set and verification set were 94.72, 90.77, and $91.84 \%$, respectively. In addition, we evaluate the prediction performance of the convolution neural network model. In the training set, test set and verification set, the sensitivity is $93.23,90.00$, and $89.97 \%$, respectively. The specificity is $95.65,91.26$, and $93.01 \%$, respectively. The accuracy is $94.72 \%$, $90.77 \%$ and $91.84 \%$, respectively. The F1 score is $0.93,0.91$, and 0.89 , respectively. The AUC (95\% CI) is 0.944 (0.936$0.952), 0.906(0.876-0.931)$ and 0.915 (0.895-0.932), respectively. The results prove that the convolutional neural network is very suitable for the prediction of dyslipidemia of steel workers and has high accuracy.

Finally, we compared the predictive performance of the convolutional neural network with the logistics model and BP neural network model of common models of dyslipidemia. We found that the predictive performance of the convolutional neural network model was better than that of the Logistics regression model and $\mathrm{BP}$ neural network model in the risk prediction of dyslipidemia of steel workers.

In the current study, the convolutional neural network model can accurately predict the risk of dyslipidemia in steel workers, and is superior to some existing predictive models of dyslipidemia. Therefore, the convolutional neural network model 
can be used to predict the risk of dyslipidemia in steel workers, and provide a basis for the formulation of early prevention strategies for dyslipidemia in steel workers, so as to improve the health status and quality of life of steel workers. In this paper, we only use the traditional convolutional neural network algorithm. So in the future, we will further study new algorithms to improve the predictive performance of the model.

\section{DATA AVAILABILITY STATEMENT}

The datasets presented in this article are not readily available because of moral restrictions. Requests to access the datasets should be directed to the corresponding author.

\section{ETHICS STATEMENT}

This research was approved by the Ethics Committee of North China University of Science and Technology. The participants provided their written informed consent to participate in this study.

\section{REFERENCES}

Arun, P. V., Buddhiraju, K. M., and Porwal, A. (2018). CNN based sub-pixel mapping for hyperspectral images. Neurocomputing 311, 51-64. doi: 10.1016/j. neucom.2018.05.051

Chauhan, A., Anand, T., Kishore, J., Danielsen, T. E., and Ingle, G. K. (2014). Occupational hazard exposure and general health profile of welders in rural Delhi. Indian J. Occup. Environ. Med. 18, 21-26. doi: 10.4103/0019-5278. 134953

Hedén Stahl, C., Novak, M., Hansson, P. O., Lappas, G., Wilhelmsen, L., and Rosengren, A. (2014). Incidence of Type 2 diabetes among occupational classes in Sweden: a 35-year follow-up cohort study in middle-aged men. Diabet. Med. 31, 674-680. doi: 10.1111/dme.12405

Hendrani, A. D., Adesiyun, T., Quispe, R., Jones, S. R., Stone, N. J., Blumenthal, R. S., et al. (2016). Dyslipidemia management in primary prevention of cardiovascular disease: current guidelines and strategies. World J. Cardiol. 8, 201-210. doi: 10.4330/wjc.v8.i2.201

Horiuchi, Y., Aoyama, K., Tokai, Y., Hirasawa, T., Yoshimizu, S., Ishiyama, A., et al. (2019). Convolutional neural network for differentiating gastric cancer from gastritis using magnified endoscopy with narrow band imaging. Dig. Dis. Sci. 65, 1355-1363. doi: 10.1007/s10620-01905862-6

Iqbal, S., Ghani, M. U., Saba, T., and Rehman, A. (2018). Brain tumor segmentation in multi-spectral MRI using convolutional neural networks (CNN). Microsc. Res. Tech. 81, 419-427. doi: 10.1002/jemt.22994

Joint committee for guideline revision (2018). 2016 Chinese guidelines for the management of dyslipidemia in adults. J. Geriatr. Cardiol. 15, 1-29. doi: 10. 11909/j.issn.1671-5411.2018.01.011

Keshari, R., Vatsa, M., Singh, R., and Noore, A. (2018). "Learning structure and strength of cnn filters for small sample size training," in Proceedings of the 2018 IEEE/CVF Conference on Computer Vision and Pattern Recognition (CVPR), Piscataway, NJ.

Lee, J. H., Kim, D. H., Jeong, S. N., and Choi, S. H. (2018). Diagnosis and prediction of periodontally compromised teeth using a deep learning-based convolutional neural network algorithm. J. Periodont. Implant Sci. 48, 114-123. doi: 10.5051/ jpis.2018.48.2.114

Lee, J. S., Chang, P. Y., Zhang, Y., Kizer, J. R., Best, L. G., and Howard, B. V. (2017). Triglyceride and HDL-C dyslipidemia and risks of coronary heart disease and ischemic stroke by glycemic dysregulation status: the strong heart study. Diabetes Care 40, 529-537. doi: 10.2337/dc16-1958

\section{AUTHOR CONTRIBUTIONS}

HW and SQ contributed to conception and design of the study. JW and JL organized the database. HW and HL performed the statistical analysis. SQ wrote the first draft of the manuscript. ZC, CL, and JJW wrote sections of the manuscript. JY contributed to manuscript revision. All authors agreed to submit this article.

\section{FUNDING}

This work was supported by the National Key R\&D Program of China (No. 2016YFC0900605).

\section{ACKNOWLEDGMENTS}

The authors thank all the participants in this study and all the members who participated in the baseline data collection.

Lin, W., Tong, T., Gao, Q., Guo, D., Du, X., Yang, Y., et al. (2018). Convolutional neural networks-based MRI image analysis for the Alzheimer's disease prediction from mild cognitive impairment. Front. Neurosci. 12:777. doi: 10. 3389/fnins.2018.00777

Liu, M. M., Wen, L., Liu, Y. J., Cai, Q., Li, L. T., and Cai, Y. M. (2018). Application of data mining methods to improve screening for the risk of early gastric cancer. BMC Med. Inform. Decis. Mak. 18:121. doi: 10.1186/s12911-018-0 689-4

Miller, M. (2009). Dyslipidemia and cardiovascular risk: the importance of early prevention. QJM 102, 657-667. doi: 10.1093/qjmed/hc p065

Ni, W. Q., Liu, X. L., Zhuo, Z. P., Yuan, X. L., Song, J. P., Chi, H. S., et al. (2015). Serum lipids and associated factors of dyslipidemia in the adult population in Shenzhen. Lipids Health Dis. 14:71. doi: 10.1186/s12944-015-0 073-7

Pereira, L. P., Sichieri, R., Segri, N. J., da Silva, R. M., and Ferreira, M. G. (2015). Self-reported dyslipidemia in central-west Brazil: prevalence and associated factors. Cien Saude Colet. 20, 1815-1824. doi: 10.1590/1413-81232015206. 16312014

Pikula, A., Beiser, A. S., Wang, J., Himali, J. J., Kelly-Hayes, M., Kase, C. S., et al. (2015). Lipid and lipoprotein measurements and the risk of ischemic vascular events: framingham study. Neurology 84, 472-479. doi: 10.1212/WNL. 0000000000001202

Qi, L., Ding, X., Tang, W., Li, Q., Mao, D., and Wang, Y. (2015). Prevalence and risk factors associated with dyslipidemia in chongqing, China. Int. J. Environ. Res. Public Health 12, 13455-13465. doi: 10.3390/ijerph12101 3455

Roth, G. A., Johnson, C., Abajobir, A., Abd-Allah, F., Abera, S. F., Abyu, G., et al. (2017). Global, regional, and national burden of cardiovascular diseases for 10 causes, 1990 to 2015. J. Am. Coll. Cardiol. 70, 1-25. doi: 10.1016/j.jacc.2017.04. 052

Stevens, W., Peneva, D., Li, J. Z., Liu, L. Z., Liu, G., Gao, R., et al. (2016) Estimating the future burden of cardiovascular disease and the value of lipid and blood pressure control therapies in China. BMC Health Serv. Res. 16:175. doi: 10.1186/s12913-016-1420-8

Tong, J., Wang, Y., Yuan, J., Yang, J., Wang, Z., Zheng, Y., et al. (2017). Effect of interaction between noise and A1166C site of AT1R Gene polymorphism on essential hypertension in an iron and steel enterprise workers. J. Occup. Environ. Med. 59, 412-416. doi: 10.1097/JOM.00000000000 00970 
Wang, C., Li, Y., Wang, L., Li, L., Guo, Y., Zhang, L., et al. (2012). Development and evaluation of a simple and effective prediction approach for identifying those at high risk of dyslipidemia in rural adult residents. PLoS One 7:e43834. doi: 10.1371/journal.pone.0043834

Wu, J., Li, J., Wang, J., Zhang, L., and Yuan, J. (2019a). Risk prediction of type 2 diabetes in steel workers based on convolutional neural network. Neural Comput. Appl. 3, 1-16.

Wu, J., Wei, W., Zhang, L., Wang, J., Robertas, D., Li, J., et al. (2019b). Risk assessment of hypertension in steel workers based on LVQ and fisher-SVM deep excavation. IEEE Access. 7, 23109-23119. doi: 10.1109/access.2019.289 9625

Xu, R., Chen, T., Xia, Y., Lu, Q., Liu, B., and Wang, X. (2015). Word embedding composition for data imbalances in sentiment and emotion classification. Cognit. Comput. 7, 226-240. doi: 10.1007/s12559-0159319-y

Yang, X., Xu, C., Wang, Y., Cao, C., Tao, Q., Zhan, S., et al. (2018). Risk prediction model of dyslipidaemia over a 5-year period based on the Taiwan MJ health check-up longitudinal database. Lipids Health Dis. 17:259. doi: 10.1186/s12944018-0906-2
Yao, L., Zhong, Y., Wu, J., Zhang, G., Chen, L., Guan, P., et al. (2019). Multivariable logistic regression and back propagation artificial neural network to predict diabetic retinopathy. Diabetes Metab. Syndr. Obes. 12, 1943-1951. doi: 10.2147/ DMSO.S219842

Zhang, X., Tang, F., Ji, J., Han, W., and Lu, P. (2019). Risk prediction of dyslipidemia for chinese han adults using random forest survival model. Clin. Epidemiol. 11, 1047-1055. doi: 10.2147/CLEP.S223694

Conflict of Interest: The authors declare that the research was conducted in the absence of any commercial or financial relationships that could be construed as a potential conflict of interest.

Copyright (C) 2020 Wu, Qin, Wang, Li, Wang, Li, Chen, Li, Wang and Yuan. This is an open-access article distributed under the terms of the Creative Commons Attribution License (CC BY). The use, distribution or reproduction in other forums is permitted, provided the original author(s) and the copyright owner(s) are credited and that the original publication in this journal is cited, in accordance with accepted academic practice. No use, distribution or reproduction is permitted which does not comply with these terms. 